\title{
Outcomes of Mechanical Thrombectomy in Patients with Large Diffusion-Weighted Imaging Lesions
}

\author{
Yong-Hwan Cho, ${ }^{1,2}$ Jae Hyung Choi ${ }^{1,2}$ \\ Busan Regional Cerebrovascular Center,' Dong-A University Hospital, Busan, Korea \\ Department of Neurosurgery, ${ }^{2}$ Dong-A University Hospital, Dong-A University College of Medicine, Busan, Korea
}

Objective : Despite many advancements in endovascular treatment, the benefits of mechanical thrombectomy (MT) in patients with large infarctions remain uncertain due to hemorrhagic complications. Therefore, we aimed to investigate the efficacy and safety of recanalization via MT within 6 hours after stroke in patients with large cerebral infarction volumes $(>70 \mathrm{~mL})$.

Methods : We retrospectively reviewed the medical data of 30 patients with large lesions on initial diffusion-weighted imaging $(>70$ $\mathrm{mL}$ ) who underwent MT at our institution within 6 hours after stroke onset. Baseline data, recanalization rate, and 3-month clinical outcomes were analyzed. Successful recanalization was defined as a modified treatment in cerebral ischemia score of $2 \mathrm{~b}$ or 3 .

Results : The recanalization rate was $63.3 \%$, and symptomatic intracerebral hemorrhage occurred in six patients (20\%). The proportion of patients with modified Rankin Scale (mRS) scores of 0-3 was significantly higher in the recanalization group than in the non-recanalization group ( $47.4 \%$ vs. 9.1\%, $p=0.049)$. The mortality rate was higher in the non-recanalization group, this difference was not significant ( $15.8 \%$ vs. $36.4 \%, p=0.372$ ). In the analysis of 3-month clinical outcomes, only successful recanalization was significantly associated with $\mathrm{mRS}$ scores of $0-3(90 \% \mathrm{vs} .50 \%, p=0.049)$. The odds ratio of recanalization for favorable outcomes (mRS 0-3) was 9.00 (95\% confidence interval, $0.95-84.90 ; p=0.055$ ).

Conclusion : Despite the risk of symptomatic intracerebral hemorrhage, successful recanalization via MT 6 hours after stroke may improve clinical outcomes in patients with large vessel occlusion.

Key Words : Cerebral infarction · Ischemia $\cdot$ Mechanical thrombectomy $\cdot$ Stroke $\cdot$ Magnetic resonance imaging, diffusion weighted.

\section{INTRODUCTION}

Acute ischemic stroke caused by large vessel occlusion is associated with high mortality and poor neurological outcomes $^{18,20)}$. While intravenously administered tissue plasminogen activator (t-PA) has been shown to improve clinical outcomes among patients experiencing ischemic stroke ${ }^{11)}$, its impact on large vessel occlusion remains controversial ${ }^{17}$. Advancements in mechanical thrombectomy (MT) have increased the recanalization rate and expanded the therapeutic window in patients with large vessel occlusion ${ }^{4,15,19)}$. Two recent randomized controlled trials reported that the therapeutic time window for MT can range from 16 to 24 hours in select patients ${ }^{1,13}$. Despite these developments, the benefit of MT

- Received : March 10, 2021 •Revised : April 19, 2021 •Accepted : April 21, 2021

- Address for reprints : Jae Hyung Choi

Department of Neurosurgery, Busan-Ulsan Regional Cardiocerebrovascular Center, Dong-A University Hospital, Dong-A University College of Medicine, 32 Daesingongwonro, Seo-gu, Busan 49202, Korea

Tel : +82-51-240-5241, Fax : +82-51-242-6714, E-mail : nschoi1018@dau.ac.kr or nail1018@hanmail.net, ORCID : https://orcid.org/0000-0003-1173-2787

This is an Open Access article distributed under the terms of the Creative Commons Attribution Non-Commercial License (http://creativecommons.org/licenses/by-nc/4.0) which permits unrestricted non-commercial use, distribution, and reproduction in any medium, provided the original work is properly cited. 
in patients with large infarction volumes remains uncertain. Reperfusion of large lesions on initial diffusion-weighted imaging (DWI) is associated with hemorrhagic transformation, which can result in poor outcomes ${ }^{4-6,9,12)}$. Indeed, Yoo et al. ${ }^{21)}$ reported that all six patients with DWI lesions larger than 70 $\mathrm{mL}$ experienced poor outcomes despite a 50\% recanalization rate. However, another registry study reported that favorable outcomes were achieved in every third patient with DWI lesions larger than $70 \mathrm{~mL}$ after successful endovascular recanalization $^{3)}$. Similarly, in a previous study conducted at our institution, recanalization was associated with a modified Rankin Scale (mRS) score of $0-3$ in patients with DWI lesions $\geq 60$ $\mathrm{mL}$ who had received intravenous $\mathrm{t}-\mathrm{PA}^{10)}$. These results indicate that recanalization may benefit patients with large DWI lesions.

In the present study, we aimed to investigate the efficacy and safety of large vessel recanalization via MT within 6 hours after stroke among patients with large cerebral infarction volumes on initial DWI $(>70 \mathrm{~mL})$.

\section{MATERIALS AND METHODS}

The study design was approved by the Institutional Review Board of the Dong-A University Hospital (DAUHIRB-21-060) and fulfilled all of the requirements for patient anonymity. Informed consent was waived because of the retrospective nature of the study.

\section{Patient population}

We retrospectively reviewed the medical data of patients with acute cerebral infarction who visited our stroke center between September 2015 and August 2019 (48 months). Only patients who had undergone femoral artery puncture for MT at our institution within 6 hours after stroke onset and exhibited DWI lesion volumes $>70 \mathrm{~mL}$ on initial imaging were included. Patients who were unable to undergo magnetic resonance imaging (MRI) prior to MT and those with pre-stroke mRS scores $\geq 2$ were excluded.

\section{Study variables}

Patient demographics and vascular risk factors including hypertension, diabetes mellitus, dyslipidemia, current cigarette smoking, atrial fibrillation, coronary artery disease, and previous cerebrovascular accident were recorded as baseline variables. Time intervals from stroke onset to arrival, stroke onset to groin puncture, and groin puncture to MT completion were recorded as time variables. The National Institutes of Health Stroke Scale (NIHSS) scores were obtained at admission. Occlusion site and Left hemisphere involvement were also recorded as radiologic data. Clinical outcomes were evaluated using the mRS score at 3 months after stroke by reviewing medical records.

DWI lesion volumes were estimated using a semiautomatic method, as previously described for our institution ${ }^{10)}$. All areas showing hyperintense signals were measured automatically after a line had been manually drawn along the margin of the lesion in each slice using a region-of-interest calculator of the picture archiving and communication system. The volume of the DWI lesion was then calculated as the sum of the product of the measured area in each slice and the slice thickness ${ }^{10)}$.

MT was performed by two interventionists. Recanalization after MT was graded using the modified Treatment in Cerebral Ischemia (mTICI) scale ${ }^{22)}$. Successful recanalization was defined as an mTICI score of $2 \mathrm{~b}$ or 3 .

\section{Statistical analysis}

Statistical analysis was performed using PASW ver. 18 software (SPSS, Chicago, IL, USA). Categorical data were compared using the Pearson chi-square test or Fisher's exact test. Continuous variables were evaluated using Student's t-test or Mann-Whitney U-test. Logistic regression analyses were used to estimate the contribution of the variables to the outcomes. Statistical significance was defined as $p<0.05$.

\section{RESULTS}

Among 2,971 patients who had visited our stroke center between September 2015 and August 2019 due to acute ischemic stroke, 245 underwent MT. Of these patients, only 30 exhibited DWI lesions larger than $70 \mathrm{~mL}$ on their initial MRI and were thus enrolled. The mean age of the patients in our study was $69.1 \pm 11.0$ years (range, $47-79$ ), and 18 patients (60\%) were male. The median NIHSS score was 17.5 (interquartile range [IQR], 14-20). Risk factors included hypertension in 16 patients (53.3\%), diabetes mellitus in six (20\%), smoking in 13 (43.3\%), dyslipidemia in 10 (33.3\%), coronary artery disease in 
Table 1. Characteristics of patients who underwent mechanical thrombectomy

\begin{tabular}{|c|c|c|c|c|}
\hline & \multirow{2}{*}{ Total } & \multicolumn{2}{|c|}{ Result of mechanical thrombectomy } & \multirow{2}{*}{$p$-value } \\
\hline & & mTICI 0-2a & $\mathrm{mTICl} 2 \mathrm{~b}-3$ & \\
\hline Total & $30(100.0)$ & $11(36.7)$ & $19(63.3)$ & \\
\hline \multicolumn{5}{|l|}{ Sex } \\
\hline Male & $18(60.0)$ & $8(72.7)$ & $10(52.6)$ & 0.442 \\
\hline Female & $12(40.0)$ & $3(27.3)$ & $9(47.4)$ & \\
\hline Age (years) & $69.1 \pm 11.0$ & $67.1 \pm 11.5$ & $70.2 \pm 10.8$ & 0.462 \\
\hline NIHSS score & $17.5(14-20)$ & $17(14-20)$ & $18(14-20)$ & 0.516 \\
\hline \multicolumn{5}{|l|}{ Hypertension } \\
\hline Absence & $14(46.7)$ & $3(27.3)$ & $11(57.9)$ & \\
\hline Presence & $16(53.3)$ & $8(72.7)$ & $8(42.1)$ & 0.105 \\
\hline \multicolumn{5}{|l|}{ Diabetes mellitus } \\
\hline Absence & $24(80.0)$ & $8(72.7)$ & $16(84.2)$ & \\
\hline Presence & $6(20.0)$ & $3(27.3)$ & $3(15.8)$ & 0.641 \\
\hline \multicolumn{5}{|l|}{ Smoking } \\
\hline Non-smoker & $17(56.7)$ & $5(45.5)$ & $12(63.2)$ & \\
\hline Current or ex-smoker & $13(43.3)$ & $6(54.5)$ & $7(36.8)$ & 0.454 \\
\hline \multicolumn{5}{|l|}{ Dyslipidemia } \\
\hline Absence & $20(66.7)$ & $6(54.5)$ & $14(73.7)$ & \\
\hline Presence & $10(33.3)$ & $5(45.5)$ & $5(26.3)$ & 0.425 \\
\hline \multicolumn{5}{|l|}{ CAD } \\
\hline Absence & $23(76.7)$ & $8(72.7)$ & $15(78.9)$ & \\
\hline Presence & $7(23.3)$ & $3(27.3)$ & $4(21.1)$ & 1.000 \\
\hline \multicolumn{5}{|l|}{ Atrial fibrillation } \\
\hline Absence & $11(36.7)$ & $3(27.3)$ & $8(42.1)$ & \\
\hline Presence & $19(63.3)$ & $8(72.7)$ & $11(57.9)$ & 0.466 \\
\hline \multicolumn{5}{|l|}{ Previous CVA } \\
\hline Absence & $24(80.0)$ & $9(81.8)$ & $15(78.9)$ & \\
\hline Presence & $6(20.0)$ & $2(18.2)$ & $4(21.1)$ & 1.000 \\
\hline \multicolumn{5}{|l|}{ tPA } \\
\hline Non-administration & $5(16.7)$ & $2(18.2)$ & $3(15.8)$ & \\
\hline Administration & $25(83.3)$ & 9 (81.8) & $16(84.2)$ & 1.000 \\
\hline DWI lesion & $107.55(89.7-130.5)$ & $112.4(91.5-131.2)$ & $102.7(82.5-130.3)$ & 0.672 \\
\hline \multicolumn{5}{|l|}{ Occlusion site } \\
\hline ICA & $12(40.0)$ & $4(36.4)$ & $8(42.1)$ & \\
\hline MCA & $18(60.0)$ & 7 (63.6) & $11(57.9)$ & 0.534 \\
\hline \multicolumn{5}{|l|}{ Symptomatic ICH } \\
\hline Absence & $24(80.0)$ & $8(72.7)$ & $16(84.2)$ & \\
\hline Presence & $6(20.0)$ & $3(27.3)$ & $3(15.8)$ & 0.641 \\
\hline OTD (minutes) & $118.5(44.8-172.3)$ & $54(33-173)$ & $131(56-168)$ & 0.497 \\
\hline OTP (minutes) & $215(128.8-275)$ & $140(101-275)$ & $230(155-275)$ & 0.420 \\
\hline PTF (minutes) & $85(60-110)$ & $105(85-120)$ & $65(55-100)$ & 0.018 \\
\hline \multicolumn{5}{|l|}{ Surgery } \\
\hline None & $23(76.7)$ & $9(81.8)$ & 14 (73.7) & \\
\hline Underwent & 7 (23.3) & $2(18.2)$ & $5(26.3)$ & 1.000 \\
\hline
\end{tabular}

Values are presented as mean \pm standard deviation, median (interquartile range), or number (\%). mTICI : modified Treatment in Cerebral Ischemia, NIHSS : National Institutes of Health Stroke Scale, CAD : coronary artery disease, CVA : cerebrovascular accident, tPA : tissue plasminogen activator, DWI : diffusion-weighted imaging, ICA : internal carotid artery, MCA : middle cerebral artery, ICH : intracerebral hemorrhage, OTD : onset to door time, OTP : onset to puncture time, PTF : puncture to finish time 
seven (23.3\%), atrial fibrillation in 19 (63.3\%), and previous cerebrovascular accident in six (20\%; ischemic stroke, six patients). Twenty-five patients (83.3\%) were treated with intravenous t-PA. The median volume of DWI lesions was $107.55 \mathrm{~mL}$ (IQR, 89.7-130.5). Symptomatic intracerebral hemorrhage (sICH) occurred in six patients (20\%). Total seven patients underwent additional surgery. Six patients underwent decompressive craniectomy due to malignant brain swelling. The other patient underwent neuro navigation assisted aspiration for sICH. The median onset to door time, onset to femoral artery puncture time, and puncture to completion time were 118.5 minutes (IQR, 44.8-172.3), 215 minutes (IQR, 128.8$275)$, and 85 minutes (IQR, 60-110), respectively. The recana-

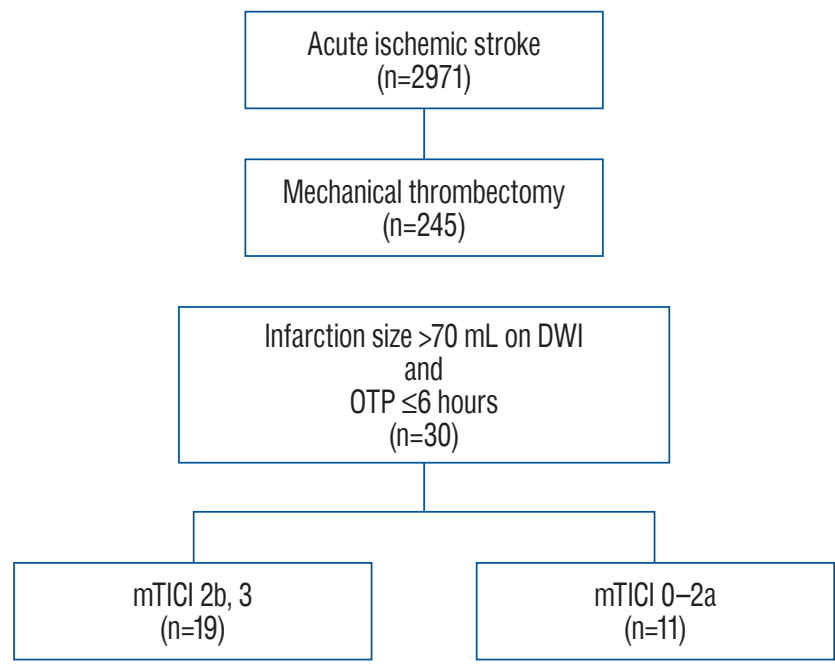

Fig. 1. Study flow chart. DWI : diffusion-weighted imaging, OTP : onset to puncture time, $\mathrm{mTICl}$ : modified Treatment in Cerebral Ischemia. lization rate was $63.3 \%$, and there were no statistically significant risk factors associated with recanalization. The rate of sICH was higher in the non-recanalization group than in the recanalization group, although this difference was not significant $(27.3 \%$ vs. $15.8 \%, p=0.641)$. There was no statistical association between additional surgery and recanalization (18.2\% vs. $26.3 \%, p=1.000)$. The total procedure time was also longer in the non-recanalization group than in the recanalization group (105 minutes [IQR, 85-120] vs. 65 minutes [IQR, 55100], $p=0.018$ ) (Table 1 and Fig. 1).

Fig. 2 shows the distribution of 3-month mRS scores for recanalization (mTICI, 2b-3) and non-recanalization (mTICI, $0-2 \mathrm{a})$ groups. Only four patients $(21.2 \%)$ in the recanalization group had mRS scores of $0-2$, whereas no patients had mRS scores of $0-2$ in the non-recanalization group. The proportion of patients with $\mathrm{mRS}$ scores of $0-3$ was also significantly higher in the recanalization group than in the non-recanalization group (47.4\% vs. 9.1\%, $p=0.049)$. Three patients died in the recanalization group (15.8\%). One patient of them died due to progressive malignant brain edema after undergoing decompressive craniectomy. The other two patient died of medical complications due to pneumonia. Four patients died in non-recanalization group (36.4\%) and all of them died due to malignant brain edema. Although the mortality rate was higher in the non-recanalization group (15.8\% vs. $36.4 \%$, $p=0.372$ ), this difference was not significant.

Table 2 shows the results of an additional analysis of the 3-month outcomes. Based on the analysis of the 3-month $\mathrm{mRS}$ score distribution, clinical outcomes were analyzed by

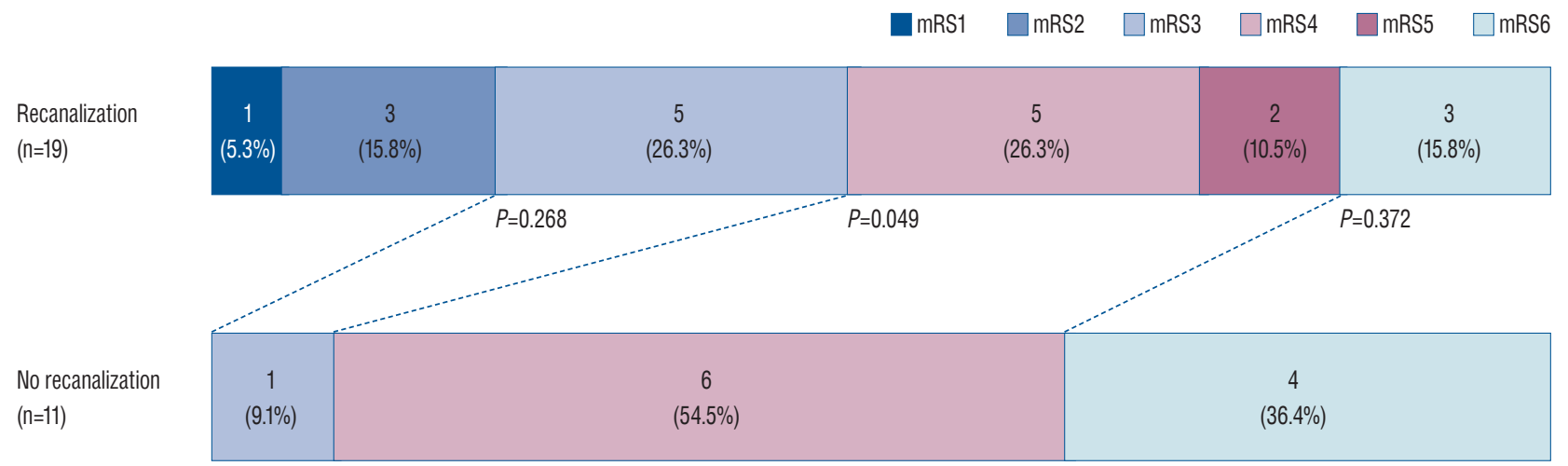

Fig. 2. Functional outcome at 3 months according to the recanalization status in patients with diffusion-weighted imaging lesions $>70 \mathrm{~mL}$. Although the proportion of patients with modified Rankin Scale (mRS) scores of 0-2 was higher in the recanalization group than in the non-recanalization group, this difference did not reach statistical significance $(21.1 \%$ vs. $0 \%, p=0.268)$. However, the proportion of patients with mRS scores of $0-3$ was also higher in the recanalization group ( $47.4 \%$ vs. $9.1 \%)$, and the difference was statistically significant $(p=0.049)$. 
Table 2. Patient characteristics according to 3-month outcomes (mRS $0-3$ vs. 4-6)

\begin{tabular}{|c|c|c|c|}
\hline & \multicolumn{2}{|c|}{ 3-month mRS score } & \multirow{2}{*}{$p$-value } \\
\hline & $0-3$ & $4-6$ & \\
\hline Total & $10(33.3)$ & $20(66.7)$ & \\
\hline \multicolumn{4}{|l|}{ Sex } \\
\hline Male & $6(60.0)$ & $12(60.0)$ & 1.000 \\
\hline Female & $4(40.0)$ & $8(40.0)$ & \\
\hline Age (years) & $67.1 \pm 11.5$ & $70.2 \pm 10.8$ & 0.286 \\
\hline NIHSS score & $16(13-20)$ & $17.5(15.3-20)$ & 0.516 \\
\hline \multicolumn{4}{|l|}{ Hypertension } \\
\hline Absence & $4(40.0)$ & $10(50.0)$ & 0.709 \\
\hline Presence & $6(60.0)$ & $10(50.0)$ & \\
\hline \multicolumn{4}{|l|}{ Diabetes mellitus } \\
\hline Absence & $9(90.0)$ & $15(75.0)$ & 0.633 \\
\hline Presence & $1(10.0)$ & $5(25.0)$ & \\
\hline \multicolumn{4}{|l|}{ Smoking } \\
\hline Non-smoker & $6(60.0)$ & $11(55.0)$ & 1.000 \\
\hline Current or ex-smoker & $4(40.0)$ & $9(45.0)$ & \\
\hline \multicolumn{4}{|l|}{ Dyslipidemia } \\
\hline Absence & $7(70.0)$ & $13(65.0)$ & 1.000 \\
\hline Presence & $3(30.0)$ & $7(35.0)$ & \\
\hline \multicolumn{4}{|l|}{ CAD } \\
\hline Absence & $8(80.0)$ & $15(75.0)$ & 1.000 \\
\hline Presence & $2(20.0)$ & $5(25.0)$ & \\
\hline \multicolumn{4}{|l|}{ Atrial fibrillation } \\
\hline Absence & $6(60.0)$ & $5(25.0)$ & 0.108 \\
\hline Presence & $4(40.0)$ & $15(75.0)$ & \\
\hline \multicolumn{4}{|l|}{ Previous CVA } \\
\hline Absence & $9(90.0)$ & $15(75.0)$ & 0.633 \\
\hline Presence & $1(10.0)$ & $5(25.0)$ & \\
\hline \multicolumn{4}{|l|}{ tPA } \\
\hline Non-administration & $2(20.0)$ & $3(15.0)$ & 1.000 \\
\hline Administration & $8(80.0)$ & $17(85.0)$ & \\
\hline DWI lesion & $95.11(75.8-117.6)$ & 114.99 (98.3-155.8) & 0.074 \\
\hline \multicolumn{4}{|l|}{ Affected hemisphere } \\
\hline Right & $6(60.0)$ & $8(40.0)$ & \\
\hline Left & $4(40.0)$ & $12(60.0)$ & 0.442 \\
\hline \multicolumn{4}{|l|}{ Symptomatic ICH } \\
\hline Absence & $10(100.0)$ & $14(70.0)$ & 0.074 \\
\hline Presence & $0(0.0)$ & $6(30.0)$ & \\
\hline \multicolumn{4}{|l|}{ Occlusion site } \\
\hline ICA & $4(40.0)$ & $8(40.0)$ & \\
\hline MCA & $6(60.0)$ & $12(60.0)$ & 1.000 \\
\hline
\end{tabular}

Table 2. Continued

\begin{tabular}{lccc}
\hline & \multicolumn{2}{c}{ 3-month mRS score } & p-value \\
\cline { 2 - 4 } & $\mathbf{0 - 3}$ & $\mathbf{4 - 6}$ & \\
\hline OTD (minutes) & $58.5(44.8-162.3)$ & $137(41.0-173.0)$ & 0.502 \\
OTP (minutes) & $195(122.5-280.0)$ & $217(132.5-275.0)$ & 0.713 \\
PTF (minutes) & $72.5(60.0-107.5)$ & $87.5(61.3-110.0)$ & 0.619 \\
Recanalization & & & \\
mTICI 0-2a & $1(10.0)$ & $10(50.0)$ & 0.049 \\
mTICI 2b, 3 & $9(90.0)$ & $10(50.0)$ & \\
Surgery & & & \\
None & $10(100.0)$ & $13(65.0)$ & \\
Underwent & $0(0.0)$ & $7(35.0)$ & 0.064 \\
\hline
\end{tabular}

Values are presented as mean \pm standard deviation, median (interquartile range), or number (\%). mRS : modified Rankin Scale, NIHSS : National Institutes of Health Stroke Scale, CAD : coronary artery disease, CVA : cerebrovascular accident, tPA : tissue plasminogen activator, DWI : diffusion-weighted imaging, ICH : intracerebral hemorrhage, ICA : internal carotid artery, MCA : middle cerebral artery, OTD : onset to door time, OTP : onset to puncture time, PTF : puncture to finish time, $\mathrm{mTICl}$ : modified Treatment in Cerebral Ischemia

dividing the patient group into the mRS $0-3$ and mRS 4-6 groups. The mRS 0-3 group exhibited a smaller DWI lesion size, less left hemisphere involvement and a lower rate of sICH than the mRS 4-6 group. However, this difference was not significant ( $p=0.074, p=0.442$, and $p=0.074$, respectively). All of the patient who underwent additional surgery were included in the mRS $4-6$ group. ( $0 \%$ vs. $35 \%, p=0.064)$. It is assumed that the patient condition such as malignant infarction or sICH that brought the surgery affected the result. Only successful recanalization was significantly associated with better outcomes ( $90 \%$ vs. $50 \%, p=0.049$ ). Simple logistic regression analysis revealed that the odds ratio for the association between recanalization and favorable outcomes was $9.00(95 \%$ confidence interval [CI], 0.95-84.90; $p=0.055)$.

\section{DISCUSSION}

The present single-center study examined the efficacy and safety of large vessel recanalization via MT within 6 hours after stroke in patients with large cerebral infarction volumes. Our analysis indicated that the proportion of patients with mRS scores of 0-3 was significantly higher in the recanalization group than in the non-recanalization group. Despite the 
small sample size of our study, our findings are in accordance with those of previous studies that have highlighted the potential benefits of MT in patients with large lesions on pretreatment $\mathrm{DWI}^{3,7,8,14)}$.

The analysis of 3-month clinical outcomes indicated that the recanalization group included a greater proportion of patients with mRS scores of $0-2$, which have been conventionally defined as favorable outcomes (20.8\% vs. $0 \%)$. Although the mortality rate was also lower in the recanalization group ( $15.8 \%$ vs. $36.4 \%)$, these differences were not statistically significant ( $p=0.268$ and $p=0.372$, respectively). Given that only four patients achieved mRS scores of $0-2$ at the 3-month follow-up, it is difficult to make comparisons by dividing patients into groups based on mRS scores of $0-2$ and 3-6. However, we observed a significant difference between the mRS $0-3$ and mRS $4-6$ subgroups ( $47.4 \%$ vs. 9.1\%, $p=0.049$ ). Therefore, we conducted an additional analysis to identify factors affecting clinical outcomes by dividing the patients into the mRS 0-3 and mRS 4-6 groups. Although mRS 0-3 is not the usual definition of a good outcome, in their study of patients with stroke exhibiting confirmed large vessel occlusion at the onset of intravenous t-PA, Rangaraju et al. ${ }^{16)}$ reported that health-related quality of life was similar in the 3-month mRS 2 and 3 groups despite differences in functional disability. Therefore, they suggested that good outcomes for moderate-to-severe ischemic stroke be defined based on mRS scores of $0-3$, rather than on scores of $0-2^{16}$. DWI lesions are generally irreversible ${ }^{2)}$, and large DWI lesions can lead to severe disability and sequelae, making it difficult for patients to perform activities of daily living (mRS, 2). Therefore, we thought that grouping them to mRS $0-3$ and mRS $4-6$ could be useful in the comparison of clinical outcomes when conventional definition was inapplicable for analysis. Similarly, other studies regarding large infarction and MT have also divided clinical outcomes based on mRS ranges of $0-3$ and $4-6^{7,8)}$.

In the present study, the recanalization rate was $63.3 \%$, while the rate of sICH was $20 \%$ (recanalization group, $15.8 \%$ vs. non-recanalization group, $27.3 \%$ ). Our analysis indicated that $47.4 \%$ of patients who experienced recanalization achieved a mRS score of $0-3$ at 3 months, which was not associated with demographic data or vascular risk factors. Although the DWI lesion area and the presence of sICH approached borderline significance ( $p=0.074$ and $p=0.074$, respectively), only successful recanalization was significantly related to favorable out- comes. Generally, previous studies of large infarction and endovascular treatment have reported that age, DWI area, and recanalization are associated with clinical outcomes ${ }^{3,8,14)}$. This discrepancy is likely explained by the small number of patients included in our study. Given that only successful recanalization fit into the logistic regression model, we conducted a simple logistic regression analysis, which yielded an odds ratio of 9.00 (95\% CI, 0.95-84.90) and a $p$ value approaching statistical significance $(p=0.055$ ). Consequently, our results suggest that successful recanalization via MT may improve the quality of life of patients with large vessel occlusion when t-PA administration has proven unsuccessful.

Generally, ICH after recanalization treatment was considered as a reperfusion syndrome ${ }^{12}$. In the present study, the rate of sICH in the non-recanalization group was higher than that in the recanalization group ( $27.3 \%$ vs. $15.8 \%, p=0.641)$. This may be explained by a greater number of recanalization attempts in the non-recanalization group, which may have increased the number of procedures and procedure time (105 minutes [IQR, 85-120] vs. 65 minutes [IQR, 55-100], $p=0.018$ ) and resulted in more intimal injuries and subsequent hemorrhagic complications such as sICH. In the clinical outcome analysis, there was no sICH in the mRS 0-3 group. All six patients with sICH were included in the mRS 4-6 group (Table 2). Taken together, when MT was performed in patients with a large infarction core, it is assumed that recanalization should be achieved with minimal MT attempts to reduce the occurrence of sICH and achieve a good outcome.

Despite a large pre-treatment infarction core, successful recanalization through MT might improve outcomes by inducing changes in the large ischemic penumbra. Kerleroux et al. ${ }^{8)}$ reported no difference in favorable outcomes between the MT and control groups in their entire population, including patients with DWI lesions $>70 \mathrm{~mL}$. However, after adjusting for the core perfusion mismatch ratio (CPMR), receiving MT was associated with an increased probability of a favorable outcome (mRS, 0-3) as CPMR increased. Significant differences were observed above a CPMR of $1.72^{8)}$. Although we did not quantify the degree of decrease in perfusion area in our study, we believe that MT may have salvaged a portion of the area, resulting in improved outcomes.

The present study has several limitations, including its single-center retrospective design, which may have led to selection bias. Moreover, some results did not achieve statistical 
significance due to the small number of patients included. While prospective randomized controlled trials are required to verify our findings, these are difficult to implement due to ethical concerns. Additional studies involving larger numbers of patients should aim to determine the efficacy and safety of recanalization via MT in patients with large infarction cores.

\section{CONCLUSION}

Despite the risk of sICH, successful large vessel recanalization could improve clinical outcomes even in high-risk patients with a large infarction core, provided that it is performed within 6-hour of onset of ischemic symptoms.

\section{CONFLICTS OF INTEREST}

No potential conflict of interest relevant to this article was reported.

\section{INFORMED CONSENT}

This type of study does not require informed consent.

\section{AUTHOR CONTRIBUTIONS}

Conceptualization : JHC

Data curation : YHC, JHC

Formal analysis : $\mathrm{YHC}$

Funding acquisition : JHC

Methodology : YHC

Project administration : JHC

Visualization : YHC, JHC

Writing - original draft : YHC

Writing - review \& editing : JHC

\section{ORCID}

Yong-Hwan Cho https://orcid.org/0000-0002-0840-0039 Jae Hyung Choi https://orcid.org/0000-0003-1173-2787

\section{- Acknowledgements}

This work was supported by the Dong-A University research fund.

\section{References}

1. Albers GW, Marks MP, Kemp S, Christensen S, Tsai JP, Ortega-Gutierrez $S$, et al. : Thrombectomy for stroke at 6 to 16 hours with selection by perfusion imaging. N Engl J Med 378 : 708-718, 2018

2. Campbell BC, Purushotham A, Christensen S, Desmond PM, Nagakane $Y$, Parsons MW, et al. : The infarct core is well represented by the acute diffusion lesion: sustained reversal is infrequent. J Cereb Blood Flow Metab 32 : 50-56, 2012

3. Gilgen MD, Klimek D, Liesirova KT, Meisterernst J, Klinger-Gratz PP, Schroth $G$, et al. : Younger stroke patients with large pretreatment diffusion-weighted imaging lesions may benefit from endovascular treatment. Stroke $46:$ 2510-2516, 2015

4. Goyal M, Menon BK, van Zwam WH, Dippel DW, Mitchell PJ, Demchuk $A M$, et al. : Endovascular thrombectomy after large-vessel ischaemic stroke: a meta-analysis of individual patient data from five randomised trials. Lancet 387 : 1723-1731, 2016

5. Hao Y, Yang D, Wang H, Zi W, Zhang M, Geng Y, et al. : Predictors for symptomatic intracranial hemorrhage after endovascular treatment of acute ischemic stroke. Stroke 48 : 1203-1209, 2017

6. Jovin TG, Yonas H, Gebel JM, Kanal E, Chang YF, Grahovac SZ, et al. : The cortical ischemic core and not the consistently present penumbra is a determinant of clinical outcome in acute middle cerebral artery occlusion. Stroke 34 : 2426-2433, 2003

7. Kakita H, Yoshimura S, Uchida K, Sakai N, Yamagami H, Morimoto T, et al. : Impact of endovascular therapy in patients with large ischemic core: subanalysis of recovery by endovascular salvage for cerebral ultra-acute embolism japan registry 2. Stroke 50 : 901-908, 2019

8. Kerleroux B, Janot K, Dargazanli C, Daly-Eraya D, Ben-Hassen W, Zhu F, et al. : Perfusion imaging to select patients with large ischemic core for mechanical thrombectomy. J Stroke 22 : 225-233, 2020

9. Kimura K, Iguchi Y, Shibazaki K, Terasawa Y, Inoue T, Uemura J, et al. : Large ischemic lesions on diffusion-weighted imaging done before intravenous tissue plasminogen activator thrombolysis predicts a poor outcome in patients with acute stroke. Stroke 39 : 2388-2391, 2008

10. Nah HW, Kim DH, Kang M, Choi JH, Park HS, Cha JK : Thrombolysis in large diffusion-weighted imaging lesions: lower chance but still a chance. J Stroke Cerebrovasc Dis 27 : 1511-1516, 2018

11. National Institute of Neurological Disorders and Stroke rt-PA Stroke Study Group : Tissue plasminogen activator for acute ischemic stroke. N Engl J Med 333 : 1581-1587, 1995

12. Nawabi J, Kniep H, Schön G, Flottmann F, Leischner $H$, Kabiri $R$, et al. : Hemorrhage after endovascular recanalization in acute stroke: lesion extent, collaterals and degree of ischemic water uptake mediate tissue 
vulnerability. Front Neurol $10:$ 569, 2019

13. Nogueira RG, Jadhav AP, Haussen DC, Bonafe A, Budzik RF, Bhuva P, et al. : Thrombectomy 6 to 24 hours after stroke with a mismatch between deficit and infarct. N Engl J Med 378 : 11-21, 2018

14. Panni P, Gory B, Xie Y, Consoli A, Desilles JP, Mazighi M, et al. : Acute stroke with large ischemic core treated by thrombectomy. Stroke 50 : 1164-1171, 2019

15. Penumbra Pivotal Stroke Trial Investigators: The penumbra pivotal stroke trial: safety and effectiveness of a new generation of mechanical devices for clot removal in intracranial large vessel occlusive disease. Stroke 40 : 2761-2768, 2009

16. Rangaraju S, Haussen D, Nogueira RG, Nahab F, Frankel M : Comparison of 3-month stroke disability and quality of life across modified rankin scale categories. Interv Neurol 6 : 36-41, 2017

17. Sawyer RN : Intravenous tissue plasminogen activator for large vessel ischemic stroke - is there still a role? Neurosurgery 85(suppl_1) : S34-S37, 2019

18. Smith WS, Lev MH, English JD, Camargo EC, Chou M, Johnston SC, et al. : Significance of large vessel intracranial occlusion causing acute ischemic stroke and TIA. Stroke $40:$ 3834-3840, 2009

19. Smith WS, Sung G, Starkman S, Saver JL, Kidwell CS, Gobin YP, et al. : Safety and efficacy of mechanical embolectomy in acute ischemic stroke: results of the MERCI trial. Stroke 36 : 1432-1438, 2005

20. Smith WS, Tsao JW, Billings ME, Johnston SC, Hemphill JC 3rd, Bonovich DC, et al. : Prognostic significance of angiographically confirmed large vessel intracranial occlusion in patients presenting with acute brain ischemia. Neurocrit Care 4 : 14-17, 2006

21. Yoo AJ, Verduzco LA, Schaefer PW, Hirsch JA, Rabinov JD, González RG : MRI-based selection for intra-arterial stroke therapy: value of pretreatment diffusion-weighted imaging lesion volume in selecting patients with acute stroke who will benefit from early recanalization. Stroke 40 : 2046-2054, 2009

22. Zaidat 00, Yoo AJ, Khatri P, Tomsick TA, von Kummer R, Saver JL, et al. : Recommendations on angiographic revascularization grading standards for acute ischemic stroke: a consensus statement. Stroke 44 : 26502663, 2013 